TRAM flap reconstruction is traditionally associated of severe pain, requiring opioid analgesia, with all known side effects.

This case reports an opioid-free anesthesia for TRAM flap reconstruction, using ESPB.

Methods Female, 53-years, ASA III, with hypertension, obesity and SAOS, admitted for TRAM flap reconstruction.

Bilateral ESPB was performed, under ultrasound guidance. It was injected $25 \mathrm{ml}$ of ropivacaine $0,375 \%(2.6 \mathrm{mg} / \mathrm{kg})$ and dexamethasone $4 \mathrm{mg}$ on each side, at T4 level.

Under ASA standard and invasive blood pressure monitoring, a totally intravenous general anesthesia was maintained with propofol and ketamine.

Acetaminophen and ketorolac were administered 30 minutes before end of surgery. No complications recorded during intra-operative period and patient emerged comfortable from anesthesia.

Results On PACU, patient remained comfortable with maximum pain of $1 / 10$ on NRS, without need of additional analgesics.

Postoperative analgesia consisted of acetaminophen and ketorolac every 8 hours and, during first 2 days, the worst pain recorded was 3/10, without need of opioid analgesia.

No complications of the ESPB was recorded and patient was discharge home after 4 days.

Conclusions ESPB is useful, easy and fast strategy that may be used as a valuable adjunct for postoperative analgesia in TRAM flap reconstruction, which pose a challenge in pain control.

Moreover, it offers an advantage in terms of reducing opioid requirements contributing for enhanced recovery.

\section{THE APPLICATION OF A COMBINATION OF INTERSCALENE AND PARAVERTEBRAL BLOCKS IN A PATIENT WITH A PATHOLOGICAL HUMERUS FRACTURE: A CASE REPORT}

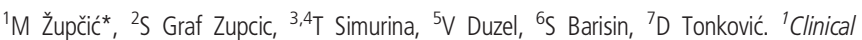
Hospital Centre Rijeka, Clinic of Anesthesiology and Intensive Care Medicine, University of Rijeka, Faculty of Medicine, Rijeka, Croatia; '2University of Rijeka, Faculty of Medicine, Hospital Centre Rijeka, Clinic of Neurology, Rijeka, Croatia; ${ }^{3}$ J. J. Strossmayer University, Faculty of Medicine Osijek, General Hospital Zadar, Department of Anesthesiology and Intensive Care Medicine, Zadar, Croatia; ${ }^{4}$ University of Zadar, Department of Health Studies, Zadar, Croatia; ${ }^{5}$ Fiona Stanley Hospital, Department of Anesthesiology, Perth, Australia; ${ }^{6}$ Clinical Hospital Dubrava, Clinic of Anesthesiology and Intensive Care Medicine, J. J. Strossmayer University, Faculty of Medicine, Zagreb, Croatia; ${ }^{7}$ School of Medicine, University of Zagreb, Clinical Hospital Centre Zagreb, Clinic of Anesthesiology, Reanimatology and Intensive Care Medicine, Zagreb, Croatia

10.1136/rapm-2021-ESRA.181

Background and Aims Most amputation procedures at the shoulder joint, on patients suffering from pathological humerus fractures are performed under general anesthesia.Here we show a case of an American Society of Anesthesiologists classification (ASA) III patient, scheduled for amputation of the humerus, at the shoulder joint. Due to a high risk procedure under general anesthesia, we decided to apply interscalene and paravertebral blocks along with intravenous sedation.

Methods A 55-year-old male, ASA III patient, was scheduled for amputation of his right humerus. The patient had a history of bladder cancer with multiple metastases on the lungs, lymph nodes and bones. He also suffered from a pathological fracture of left humerus, with presence of left-hand cellulitis.
During preparation for surgery, an invasive blood pressure measurement was set up, while the interscalene and paravertebral spaces were identified using a nerve-stimulating needle and a linear ultrasound probe of 8 and 12 Hertz. An anesthetic solution of $0.5 \%$ levobupivacaine was applied at Thoracic (Th) 2 and Th3 levels (5 milliliters per level) and to the brachial plexus (20 milliliters). We used 1\% lidocaine for skin infiltration and sedation was performed with a continuous infusion of $1 \%$ propofol.

Results Sensory blockade occurred after 18 minutes and lasted for about 16 hours in the shoulder and 10 hours in the axilla region, with stable hemodynamic parameters and no perioperative complications.

Conclusions Such precise administration of small doses of long-acting local anesthetic at multiple levels has resulted in a satisfactory anesthesia and analgesia without hemodynamic and respiratory complications.

\section{A RETROSPECTIVE COHORT STUDY OF BRACHIAL PLEXUS BLOCKS IN VASCULAR SURGERIES FOR HEMODIALYSIS ACCESS OF KIDNEY PATIENTS IN A TERTIARY CARE CENTER (2016-2019)}

A Rosales*, N Aypa. Makati Medical Center, Makati, Philippines

\subsection{6/rapm-2021-ESRA.182}

Background and Aims Brachial plexus blocks (BPB) have been used to provide surgical anesthesia in vascular procedures. Regional anesthesiologists must correlate technique with the procedure to establish protocols in performing peripheral nerve blocks in renal patients for hemodialysis. The primary objective of this study is to describe relationship between BPB used and the type of surgery done for hemodialysis access.

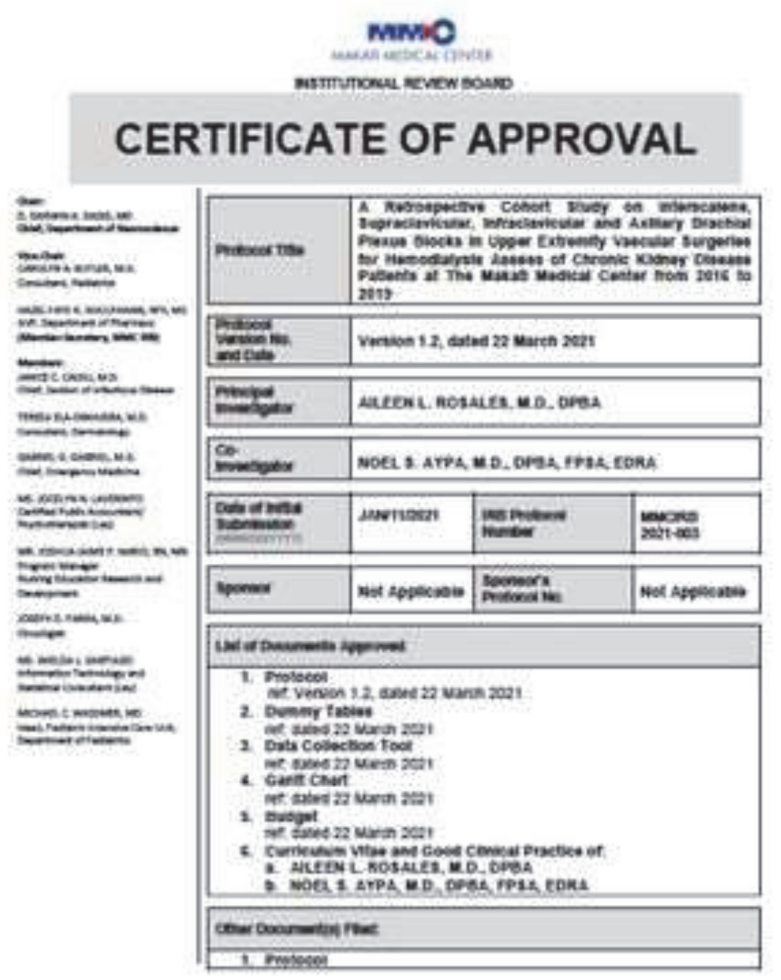

Abstract 182 Figure 1 


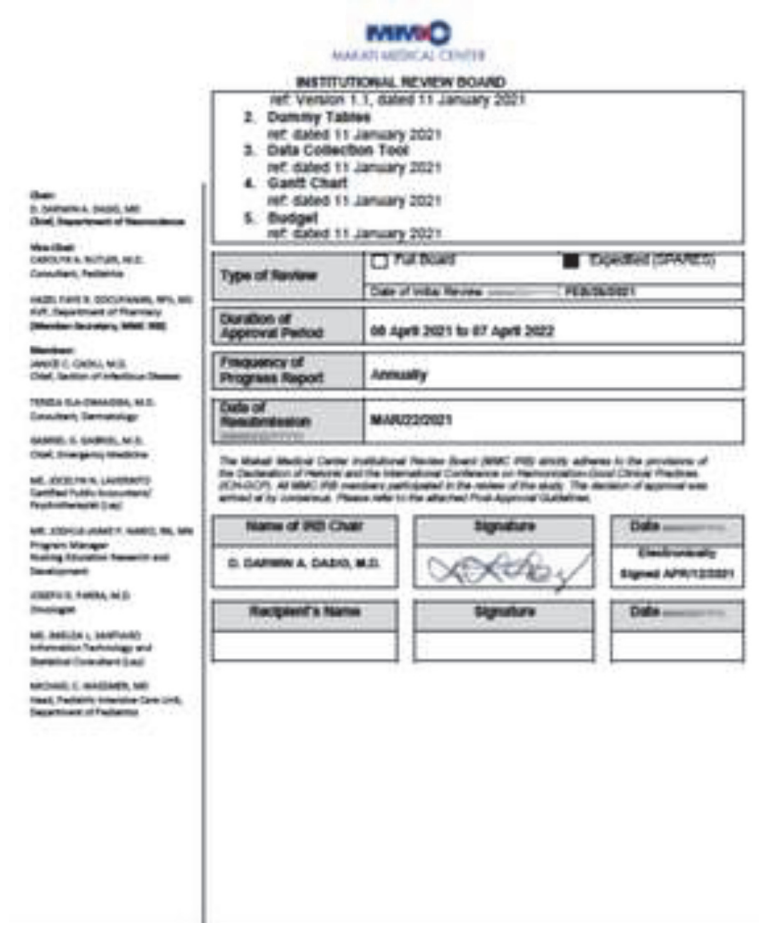

Abstract 182 Figure 2

Methods This was a retrospective, analytical, observational, cohort type of study conducted through chart review of kidney patients at a tertiary care center approved by the IRB (Protocol Number 2021-003).

Results A total of 355 patients were included in this study where the median age was 64 years old. More than half of the cohort (57.18\%) was male. Most of the subjects (93\%) were classified as ASA category III. The most common surgical procedure for securing vascular access was arteriovenous fistula creation (39.72\%) while the most common BPB performed was axillary approach (59.15\%), followed by supraclavicular approach (19.44\%), infraclavicular approach (14.93\%) and interscalene approach (6.48\%).

All four techniques recorded no incidence of inadequate block and only low incidence for need of rescue pain medication for infraclavicular $(0.47,95 \%$ CI: $0-10.01)$ and axillary (0.47, 95\% CI: 0-2.62) blocks. This resulted to a low overall incidence of need of rescue pain medication at $0.56,95 \% \mathrm{CI}$ : $0-2.02$.

Conclusions Axillary, infraclavicular and supraclavicular BPB are appropriate in procedures involving the wrist, distal arm and elbow (i.e., AV fistula creation). In procedures involving the arm such as AV graft creation and thrombectomy, infraclavicular, supraclavicular and interscalene BPB may be utilized.

\section{A CASE REPORT OF ERECTOR SPINAE PLANE BLOCK WITH CONTINUOUS INFUSION FOR MULTIPLE RIB FRACTURES}

J Cleland*, M Hulgur. Royal Albert Edward Infirmary, Wigan, UK

10.1136/rapm-2021-ESRA. 183
Background and Aims Rib fractures cause significant pain and respiratory complications ${ }^{1}$. Common analgesic techniques include opiates and thoracic epidurals, both have significant side effects ${ }^{2}$. Erector spinae plane (ESP) block with catheter insertion has been used as an alternative due to its ability to provide analgesia with a potentially better side effect profile ${ }^{3,4}$. We describe a case of a 74 year old gentleman with a significant smoking history and COPD who had suffered trauma and fractured ribs $8-11$ on his right side.

Methods To perform the ESP block we injected local anaesthetic and then inserted a catheter between the erector spinae muscle and transverse processes (figure 1). A bolus dose of 30 $\mathrm{ml} 0.25 \%$ levobupivacaine was injected followed by infusion of bupivacaine $0.125 \% 10 \mathrm{ml} / \mathrm{hr}$. This was later increased to $15 \mathrm{ml} / \mathrm{hr}$ due to some patient discomfort.

Results Prior to the ESP block the patient had required 44 PCA doses in $24 \mathrm{hrs}$, afterwards he required 10 doses in 24 hrs. Pain scores improved from a baseline level of $8 / 10$ with episodes of $10 / 10$ prior to block, to a baseline level of $0 / 10$ with episodes of $2 / 10$ afterwards. Prior to ESP block he was unable to use the incentive spirometer due to pain, the following day he was able to use the incentive spirometer without any pain (figure 2).

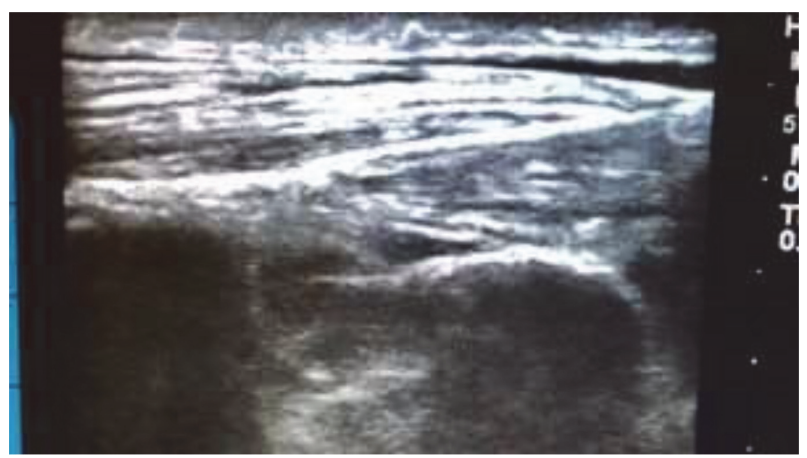

Abstract 183 Figure 1

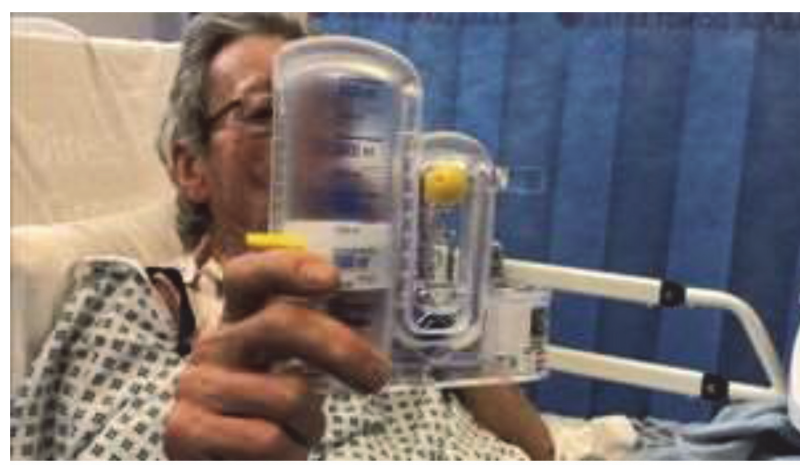

Abstract 183 Figure 2

Conclusions The ESP block both reduced the patients pain symptoms and improved his respiratory function. The patient much preferred the ESP block to the PCA he had been using before. ESP block is a useful tool in the management of pain from rib fractures. 